\title{
Using forensic handwriting principles for rapid signature screening
}

\author{
BRIAN CRAYTHORNE \\ ORCID: 0000-0002-7142-8843 \\ Forensic Science Agency of Northern Ireland \\ Carrickfergus, United Kingdom
}

\begin{abstract}
Examination of a signature is a lengthy process conducted under laboratory conditions, requiring precision and caution. When a rapid screening of signatures is required, the basic principles of forensic signature comparison can be adapted. Learning points from signature screening examinations are outlined to aid handwriting experts in carrying out similar procedures.
\end{abstract}

Keywords: signature examination, screening, best practice, comparison

This paper has been compiled from the experiences of handwriting experts carrying out rapid screening of numerous signatures to aid the election process in Northern Ireland. The details of the rapid screening procedure are detailed as follows. The questioned document is a proxy voting form bearing a questioned signature requesting a proxy vote. The specimen signatures are held on a database and may be months or years old, and range from only one signature to up to six written over a period of several years. The specimens are digital images of scanned signatures and the questioned signature is usually in the form of a copy image. A decision has to be made in a few minutes as to whether or not the signature is genuine. There should be a bias towards declaring signatures genuine - unless there is a good reason to declare them forgeries - so that 
the voter is not incorrectly denied a vote. As it is a real possibility that family members may have forged the questioned signatures, the signatures of close family members are available for comparison. The examination is carried out with no magnification equipment, but it is sometimes possible to zoom in on the digital images of the questioned signatures.

Thus the conditions are set for a rapid screening process of hundreds of signatures. In order to carry out the examination in less than ideal conditions, the handwriting expert has to draw upon certain principles of forensic handwriting examination in order to maximise the number of correct opinions whilst erring on the side of caution in order not to mistakenly deny someone a vote.

The principles applied begin with the basis that handwriting is a complex fine motor skill involving the writing of subtle strokes while at the same time incorporating from memory details of written and spoken language as well as spatial distribution. The signature is also treated as a special piece of writing, usually written by the writer and adapted to be a personal identifier.

The next principle to consider is how signatures change with the age of a person. The signatures on voting forms, which are most likely to be forged, are the signatures of the young (as they are often away from home at university and have their vote cast by their parents) and the elderly (as they are often too infirm to vote and have their vote cast by their adult children).

Once again we use the basic principle that handwriting is taught at school from a copybook and almost immediately - due to differences in mental and physical ability - the handwriting of an individual begins to divert from the copybook style. The handwriting develops during school days and can change markedly in college/university days when a lot of writing is required, and upon starting work which can require regular signing. It is at this stage that the final signature design is formed. In mid-life, the signature shows only small changes and development. Unfortunately, in later life, changes to mental and physical ability with the onset of age-related infirmities and illnesses lead to a deterioration in signature quality. 
Using this principle, one can postulate that it is possible to expect major changes in the signatures of the young - particularly the development of the signature from visual similarity to the person's handwriting to becoming more stylized, with loops and curves replacing actual structured letter designs.
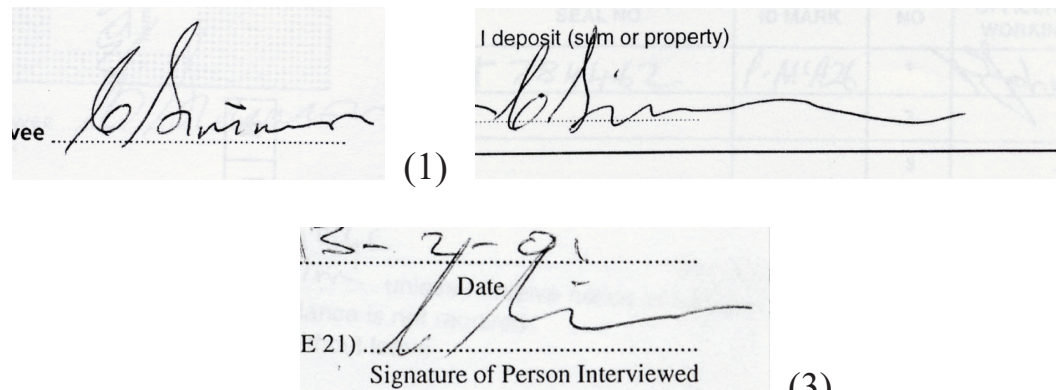

Figure 1. From left to right: the development of a signature to a more stylized form over time

So if one uses this principle to compare a recent signature of a young person against previously written specimens, one therefore should expect the signature to have developed from a more structured form to a less structured one.

Applying this particular handwriting rule to the rapid screening of signatures, it is assumed that the development of a signature is from more structured to less structured. So if the recently written questioned signature is less structured than the older specimen signatures, that could mean that the signature is genuine. However, another factor must be taken into consideration, namely if the questioned signature can be shown to have logically developed from the earlier specimen signatures. In order to do this, there need to be some features in common between the earlier specimen signatures and the recent questioned signature. In some signatures this is obvious, while for some it is not and a more detailed (although still in a matter of minutes rather than hours) investigation needs to be carried out. The examiner must therefore ask if there is some feature present in a similar structural form in the specimen signatures which is also present in the more recent questioned signature. 

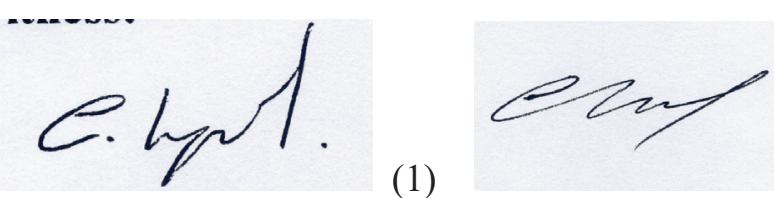

Figure 2. The loop at the top of the ' $\mathrm{C}$ ' can be used to link an earlier specimen signature (1) to the newer less structured form of the signature (2)

If we find this similarity or similarities between older specimen signatures and the newer questioned signature, then the examiner is likely to conclude that the signature is genuine.

If there are no features to tie a questioned signature to previously written specimen signatures, then there is no evidence of common authorship and the questioned signature could easily have been written by someone else attempting a generic stylized signature with little or no attempt to copy the person's genuine signature.
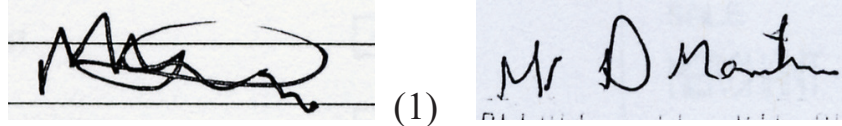

Figure 3. There is nothing to link the questioned signature (2) to the specimen (1) in terms of letter designs, and the writer of the stylized signature (1) is unlikely to revert back to

a more structured signature which is also written with a poorer artistic quality

This feature of signatures developing from structured forms to less structured stylized patterns is of particular relevance for the young writer - in his or her late teens and early twenties. When someone is older, the handwriting expert must assume the signature is relatively fixed for the purposes of rapid signature screening. What comes into play now is the principle of variability - no one writes exactly the same way every time.

So for the many signatures of people who are of "mid-life" age the examiner has to consider whether the differences between the questioned and specimen signatures are due to variation or someone forging the signature.

The overall limitation in the number of specimens hinders the handwriting expert and this would be a major problem in conventional forensic handwriting analysis. However, in the rapid screening of signatures 
the expert needs to make a decision based on what evidence can be seen from the signatures, and needs to call on his or her expertise to decide if any differences are due to variation or point to a different writer being involved. Other factors to consider are the fluency of the signature and the fact that many people signing voting forms may not sign their name on many other occasions, which can lead to greater variation between the few signatures they do write.

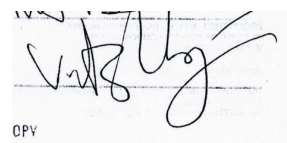

(1)

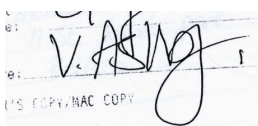

(2)

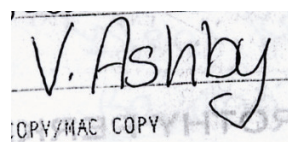

(3)

Figure 4. The consideration of variation is important with a limited number of specimen signatures

So when considering variation as well as the variation of signatures written by people who do not write very often, the expert needs to take account of factors such as whether the person wrote their name in a slightly different format or switched from upper case to lower case/ cursive letters, or vice versa.

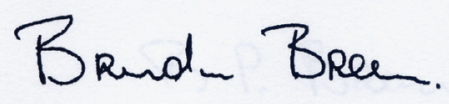

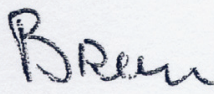

Figure 5. Another point to consider is whether the signature was written in a slightly different format

Regarding the writer's age, the questioned signatures of the elderly often provide interesting comparisons. Once more, basic principles need to be followed in order to use a consistent approach. It is taken as the most logical assumption that even if an elderly and infirm person is incapable of writing their usual healthy signature, they still try to write their normal signature to the best of their current ability. The second logical assumption is that as their handwriting is deteriorating, a signature written with a better artistic quality is to be viewed with suspicion. In normal circumstances, the handwriting expert has access to more information on the person's state of health at the time of signing, even to the details such as if they have taken medicine which momentarily improves their condition. 

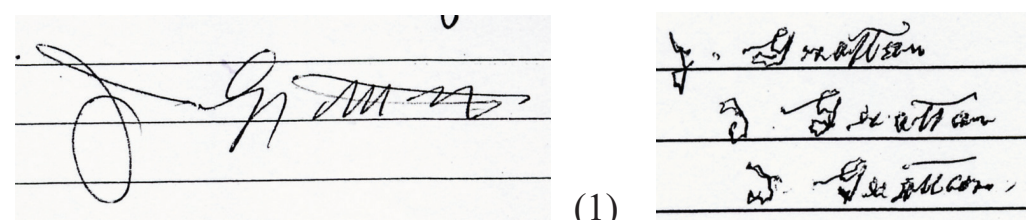

Figure 6. Signatures of the elderly - comparison of questioned (1) with specimen (2) show clear differences in fluency, handwriting ability, and letter designs

Following these principles, experts are most likely to exclude signatures that are in a different handwriting style and ones of much better artistic quality than the specimen signatures. From experience, it is fairly common for the elderly to have their vote taken by one of their children who forges their signature on the voting forms.

Fluency is an important factor in the examination of elderly people's signatures, as well as in the general examination of forged signatures. Many forged signatures as seen in rapid signature screening were written with a shaky and hesitant fluency, which marks them out as different from the specimens. Of course, the specimens are searched to see if the voter has a naturally shaky signature. It is difficult to assess other factors that the handwriting expert would normally consider as alternate hypotheses, such as: whether the voter is an alcoholic and the shakiness of the signature improves with consumption of alcohol; if the voter consumed alcohol, leading to differences in fluency and letter designs; whether the form was signed on an uneven surface or even standing up.
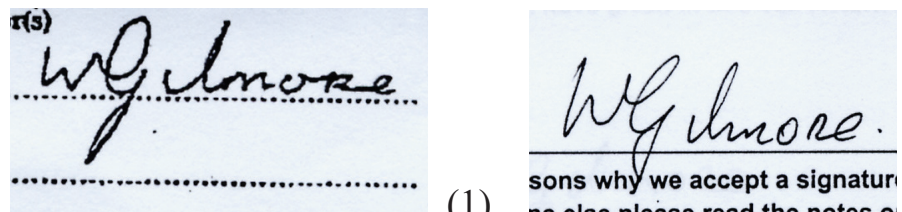

Figure 7. A difference in fluency between the questioned (1) and the specimen (2) signatures is always a cause for concern, but there is little opportunity to explore alternative hypotheses in rapid signature screening

To summarise, a marked difference in fluency would likely lead to a signature being rejected as a forgery. Likewise, a marked improvement in fluency for no apparent reason where several recent specimen 
signatures show a poor handwriting style could also very well lead to the signature being rejected. Furthermore, a complete difference in handwriting style will lead to the signature being rejected, and these are usually the easiest comparisons to carry out.

Comparison of reasonably skilful forgeries is the most difficult examination to carry out within the limited resources of rapid signature screening. With no microscope and no set of specimens showing the full range of variation of the writer, it can be difficult to spot skillful forgeries. Fortunately, many of the forgeries seen are poorly executed by people who do not write much themselves in everyday life. Poorly executed letter designs, disjointed lettering, and - of course - hesitant fluency characterise these signatures.
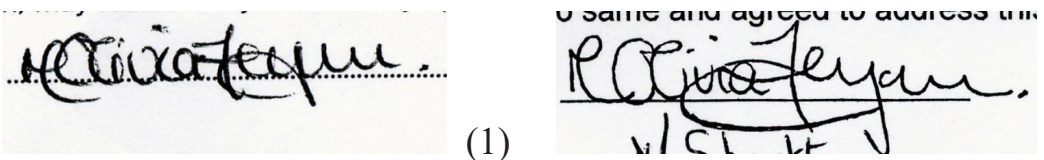

Figure 8. The conventional accomplished freehand forgery (2) is identified when compared against numerous suitable specimens (1). It is not necessarily discovered in rapid signature screening

Overwriting of a signature is something that always flags up concern to a handwriting expert. However, in rapid signature screening overwriting flags up two different possibilities, one of which is: has the overwriting been done to cover up a badly produced copied forgery? However, as the forms have to be completed in black ink, its presence written over blue ink is more likely to indicate overwriting has occurred so that the signature is in the correct colour of ink. A closer look at the signature, perhaps aided by digital zoom, should determine which scenario is the more likely one.

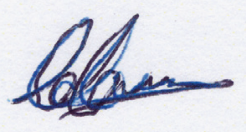

Figure 9. Overwriting is often a cause for suspicion but in cases of black/blue ink overwriting, a mistake in signing may be the explanation 
Finally, as an aid to the examination, the signatures of family members are also present on the database of specimen signatures. Thus, if a forgery is suspected, the signatures of family members are also compared against the questioned signature of the voter. Quite often it can be seen that the questioned signature more closely resembles the signature of a family member than it resembles the signature of the voter. In such cases this additional evidence aids the declaration of a forgery with greater confidence.
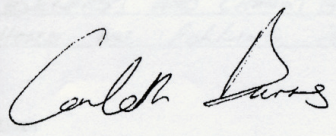

(1)

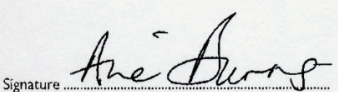

(2)

Figure 10. Comparison of the formation of the surname should be investigated against specimens of other family members

\section{Conclusions}

Rapid signature screening is different to conventional forensic handwriting comparison, but the same principles can be applied to obtain the best possible conclusion. Whilst the examination will always be a compromise, the examiner must draw upon much knowledge and expertise to make rapid decisions based upon a few signatures.

\section{References}

Hilton O., "Consideration of the writer's health in identifying signatures and detecting forgery", Journal of Forensic Sciences 11, 1969, no. 2.

Harrison W.R., Suspect documents, London 1966.

Hilton O., Scientific examination of questioned documents, New York 1982. 